\title{
The relationship between phonological and morphological deficits in Broca's aphasia: Further evidence from errors in verb inflection
}

\author{
Natalie Braber ${ }^{\mathrm{a}}$, Karalyn Patterson ${ }^{\mathrm{b}}$, Katherine Ellis ${ }^{\mathrm{a}}$, Matthew A. Lambon Ralph ${ }^{\mathrm{a}, *}$ \\ a University of Manchester, $U K$ \\ ${ }^{\mathrm{b}}$ MRC Cognition and Brain Sciences Unit, Cambridge, UK
}

Accepted 24 May 2004

Available online 10 August 2004

\begin{abstract}
A previous study of 10 patients with Broca's aphasia demonstrated that the advantage for producing the past tense of irregular over regular verbs exhibited by these patients was eliminated when the two sets of past-tense forms were matched for phonological complexity (Bird, Lambon Ralph, Seidenberg, McClelland, \& Patterson, 2003). The interpretation given was that a generalised phonological impairment was central to the patients' language deficits, including their poor performance on regular past tense verbs. The current paper provides further evidence in favour of this hypothesis, on the basis of a detailed analysis of the errors produced by these same 10 patients in reading, repetition, and sentence completion for a large number of regular, irregular, and nonce verbs. The patients' predominant error types in all tasks and for all verb types were close and distant phonologically related responses. The balance between close and distant errors varied along three continua: the severity of the patient (more distant errors produced by the more severely impaired patients); the difficulty of the task (more distant errors in sentence completion $>$ reading $>$ repetition); the difficulty of the item (more distant errors for novel word forms than real verbs). A position analysis for these phonologically related errors revealed that vowels were most likely to be preserved and that consonant onsets and offsets were equally likely to be incorrect. Critically, the patients' errors exhibited a strong tendency to simplify the phonological form of the target. These results are consistent with the notion that the patients' relatively greater difficulty with regular past tenses reflects a phonological impairment that is sensitive to the complexity of spoken forms.
\end{abstract}

(C) 2004 Elsevier Inc. All rights reserved.

Keywords: Broca's aphasia; Past tense; Verb morphology; PDP connectionism; Dual-Mechanism account

\section{Introduction}

In the study of language processing, the apparent division between regular and irregular forms, in particular with respect to verbs and their past tenses has attracted a considerable amount of attention. Indeed, it is commonly cited as a test case for theories of language processing and its neural implementation (Marslen-Wilson \& Tyler, 1997, 1998; McClelland \& Patterson, 2002; Pinker \& Ullman, 2002; Ullman, 2001b). This topic has

\footnotetext{
${ }^{*}$ Corresponding author. Fax: +44-161-275-2588.

E-mail address: matt.lambon-ralph@man.ac.uk (M.A. Lambon Ralph).
}

been examined using a variety of sources of evidence including the pattern of normal and abnormal development, functional neuroimaging studies, computational neural network models, and empirical studies of aphasic patients - of which, this study is another example.

Approximately $86 \%$ of English verbs form the past tense using the regular inflection (Plunkett \& Nakisa, 1997). The stem is transformed into the past tense by the addition of -ed to the orthographic form and one of three allophones to the phonological form. The three allophones are dependent on the stem-final phoneme: the voiced allophone [d] is added to stems ending in a voiced phoneme (e.g., stabbed, loved, and raised); the unvoiced $[t]$ is used for stems ending with unvoiced phonemes 
(e.g., stopped, laughed, and raced); and for those stems already ending with an alveolar stop, voiced or unvoiced, the syllable [Id] is added (e.g., faded, hated). The past tense for the remaining $14 \%$ of verbs are formed in a variety of ways: no change (e.g., hit $\rightarrow$ hit); vowel change (e.g., meet $\rightarrow$ met); vowel change and consonant addition (e.g., leave $\rightarrow$ left); consonant change (e.g., send $\rightarrow$ sent); and a small number of high-frequency suppletive forms (e.g., go $\rightarrow$ went). Even amongst these irregular verbs, strong regularities can be found. Within each group, there are families of verbs that form the past tense in a consistent manner (e.g., ring $\rightarrow$ rang, sing $\rightarrow$ sang; weep $\rightarrow$ wept, creep $\rightarrow$ crept $)$. In addition, the final alveolar stop that always characterises the regular past tense is also a feature of many irregular past-tense forms (e.g., mean $\rightarrow$ meant, have $\rightarrow$ had, cut $\rightarrow$ cut ).

The dual-mechanism account of verb inflection is that the organisation of the cognitive and underlying neural substrates reflects the basic distinction between regular and irregular verbs (Pinker, 1999; Ullman et al., 1997). According to this account, the past-tense forms of irregular verbs are stored in a lexicon, or an associative distributed-representational system, which is a part of declarative memory. Regular past tenses, by contrast, are formed by a rule-governed procedural system, which is applied productively to regular verbs as well as novel verb forms. When a verb needs to be expressed in the past tense, it is assumed that both mechanisms begin to operate. If the verb matches a stored form in the lexicon, then the rule-governed mechanism is inhibited and the correct irregular form is retrieved from the lexicon. For regular verbs and novel words, no match is found in the lexicon and the regular past tense is formed through the application of the rule procedure. Ullman (2001a, 1997) used neuropsychological data to argue that this cognitive-modular distinction is also reflected at the neural level. According to this hypothesis, the lexicon and declarative memory are represented in the temporal lobe, whereas morphology and grammar are supported by a procedural system based on basal ganglia-frontal cortex circuits. Consistent with this hypothesis, Ullman et al. (1997) reported a double dissociation for the past-tense forms of regular and irregular verbs across different patient types. A group of patients with Alzheimer's disease and a group of posterior aphasics had poorer performance with irregular than regular verbs. In contrast, a group of patients with Parkinson's disease demonstrated a slight difference favouring irregular over regular verbs, while an anterior aphasic patient exhibited the same difference on a much larger scale.

According to an alternative view, given the pervasive consistencies that can be found amongst the irregular verbs, the contrast between regular and irregular verbs should not be viewed in absolute terms but as a more graded difference (Joanisse \& Seidenberg, 1999; McClelland \& Patterson, 2002; Rumelhart \& McClelland, 1986). This view proposes a single system that draws on semantic and phonological knowledge, and processes all verb types. The phonological process for forming the past tense from the stem is influenced by word frequency and consistencies in the mapping between stem and past-tense forms. The phonological mapping is particularly efficient, therefore, both for high frequency verbs and for the addition of the typical -ed in regular verbs. In this account, knowledge of word meaning also contributes to the formation of the past tense for all real verbs but is differentially important for low frequency, irregular verbs given that they benefit neither from frequent usage nor from the prevailing phonological pattern characterising all the regular verbs.

By this account, the double dissociation found across different patient groups is assumed to reflect phonological and semantic influences on a single process (see Joanisse \& Seidenberg, 1999 for a computational instantiation of this theory). The double dissociation is attributed to (a) the greater reliance on word meaning for irregular verbs (Joanisse \& Seidenberg, 1999) and (b) the greater phonological complexity of the regular past tense, following from the fact that morphological regularity correlates inversely with phonological regularity (Burzio, 2002; McClelland \& Patterson, 2002). This single mechanism account predicts that poor performance with irregular verbs, especially for lower frequency items, should be associated with semantic impairment, while the relative deficit for regular verbs reported in anterior aphasic patients should be associated with phonological impairment.

Two previous sets of results have been interpreted as supporting the single system theory. Patterson, Lambon Ralph, Hodges, and McClelland (2001) studied 11 patients with semantic dementia, a condition in which progressive atrophy of the anterior, inferior temporal lobes gradually erodes meaning for words, faces, objects, and other non-verbal stimuli (Bozeat, Lambon Ralph, Patterson, Garrard, \& Hodges, 2000; Hodges, Patterson, Oxbury, \& Funnell, 1992). The patients were asked to generate the past tense of regular, irregular, and novel verbs in simple sentence frames and to indicate the correct past tense in a two-alternative forced-choice recognition version of the same experiment. The degree of semantic impairment for these same target verbs was assessed using a synonym judgement test. As predicted by the Joanisse and Seidenberg (1999) model, the patients had essentially normal performance in generating and recognising the past-tense forms of novel and regular verbs, but substantially reduced accuracy on the irregular past tense that was modulated by the frequency of the words and, critically, by the degree of the patients' semantic impairment.

Bird, Lambon Ralph, Seidenberg, McClelland, and Patterson (2003) investigated the hypothesised link between poor regular verb performance and phonological impairment in 10 patients with Broca's aphasia. These patients were selected not only to have the dysfluent and agrammatic speech typical of Broca's aphasia but also 
to demonstrate an advantage for the past tense of irregular verbs in at least two out of three production tasks (sentence completion, repetition, and reading). All 10 patients exhibited substantial impairments on a variety of tasks that require activation or manipulation of phonological representations (rhyme judgement, rhyme production, phoneme segmentation, and blending). Bird et al. (2003) performed two main experiments whose results were consistent with the notion that the patients' relatively poorer regular verb performance reflected the greater phonological complexity of these past-tense forms. First, the three production tasks were repeated using regular and irregular verbs matched for the CVC complexity of their past-tense forms (e.g., the past-tense forms "stepped" and "slept" have the same phonological structure whereas, on average in an unmatched set, there will be more phonemes and more complex combinations of phonemes in the past tense of regularly inflected verbs like blink $\rightarrow$ "blinked" than in irregular forms like think $\rightarrow$ "thought"). The previously observed irregular verb advantage disappeared under the matched conditions. The second experiment contrasted regular stem and past tense pairings (e.g., pray-prayed, press-pressed) with non-verb word pairings that had a matching phonological contrast (e.g., tray-trade, chess-chest). The Broca patients were required to make same-different judgements to each spoken word pair. In each and every case, the patient's accuracy for the verb and non-verb word pairings was almost identical - suggesting that their poor verb performance reflected their more general phonological impairment rather than a specific morphological deficit. It should be noted that in a very similar task but including reaction-time measures, Tyler et al. (2002a) and Tyler, Randall, and Marslen-Wilson (2002b) also obtained no significant difference between accuracy of judgements to verb vs. non-verb pairs, but did observe significantly slower decisions in the verb condition.

Owing to the multiple tasks and the large numbers of stimulus items per task in the study by Bird et al. (2003), the initial publication included only the accuracy data and a minimal description of the error types. The focus of the present article is the nature of the errors produced by these patients in reading, repetition, and sentence completion for regular and irregular verbs, as well as a set of phonologically matched non-words. The purpose of this error analysis was to extend our understanding of the relationship between phonological and morphological deficits.

\section{Methods}

\subsection{Participants and background assessment}

Ten non-fluent aphasic patients-with a single lefthemisphere vascular lesion participated in this study (see Table 1 for demographic information about the participants). These individuals had been originally selected (by Bird et al., 2003) on the bases (a) that they had a non-fluent, Broca-type aphasia; and (b) that although all cases were markedly impaired in both irregular and regular past tense verb production, they showed a significant advantage for the irregular past tense on a screening test of sentence completion, reading, and repetition. The materials used in this screening task were not controlled for the phonological complexity of the past-tense forms and, as already mentioned above, the irregular advantage was reduced or even entirely eliminated when the patients were subsequently tested on a new set of verbs that matched the CVC structure of the regular and irregular past-tense forms (Bird et al., 2003). The patients completed a battery of background assessments to give a profile of their general language and semantic abilities. For convenience, this information is reproduced and discussed briefly here (see Table 2). Fluency of spontaneous speech was assessed by calculating the number of words produced per minute during a description of the Cookie Theft picture (Goodglass \& Kaplan, 1983). This measure confirmed that all 10 patients had very non-fluent speech with rates ranging from 5 to 47 words per minute. This is much lower than observed in age- and education-matched control participants (for example, 96 words per minute was the slowest normal rate observed by Bird, Lambon Ralph, Patterson, \& Hodges, 2000). The patients' immediate repetition span ranged from 1 to 5 digits, and their impaired syntactic comprehension was confirmed by poor scores on the Test for the Reception of Grammar (Bishop, 1989). In addition, the patients demonstrated impaired single word production in both picture naming and reading (the latter with a pattern of phonological-deep dyslexia). On tests of single word comprehension (word-picture matching and the Pyramid and Palm Trees Test: Howard \& Patterson, 1992), the patients had normal or near-normal performance. In contrast, their severely impaired phonological processing was documented across a number of standard tests of phonological awareness.

\subsection{Materials and procedure}

The patients were asked to complete three different speech production tasks: single word reading, immediate repetition, and sentence completion. The reading and repetition assessments consisted of 504 real verbs and the same number of nonce verbs. The real verbs comprised 126 regular and 126 irregular verbs (=252 items), each presented in both its stem and past-tense forms (=504 items). The nonce verbs were created from these 504 words by substituting a single phoneme within the verb stem. Only data for the patients' ability to repeat or read past-tense forms (both words and non-words) are considered in this paper. The sentence completion 
Table 1

Patient details

\begin{tabular}{llll}
\hline Patient & Age & Sex & Aetiology/CT report \\
\hline BB & 60 & F & 1996 L CVA during operation for R acoustic neuroma \\
IJ & 61 & M & 1995 Low attenuation L frontal, temporal and parietal lobes, MCA ischaemic infarct \\
IB & 53 & F & 1990 Subarachnoid haemorrhage from L MCA aneurysm and infarct \\
DE & 64 & M & 1997 Non-haemorrhagic infarct L MCA territory \\
AB & 61 & F & 1996 Embolic CVA L MCA territory \\
MB & 83 & F & 1998 Non-haemorrhagic L frontoparietal infarct (5 months previously two small R frontal infarcts) \\
RT & 58 & M & 1987 L CVA \\
VC & 65 & F & 1998 Low attenuation L frontal and parietal lobes, ischaemic infarct \\
GN & 72 & M & 1999 L frontal infarct in MCA territory, also established low density lesion in L occipital lobe \\
JL & 46 & F & 1996 L CVA involving temporal, parietal and frontal lobe \\
\hline
\end{tabular}

Table 2

General language assessments

\begin{tabular}{|c|c|c|c|c|c|}
\hline Test & Max score & Mean & $S D$ & Min & Max \\
\hline Digit span & & 3 & 1.15 & 1 & 5 \\
\hline Spontaneous speech (words per min) & & 24 & 13.90 & 5 & 47 \\
\hline TROG $^{\mathrm{a}}$ & 120 & 9.9 & 4.23 & 5 & 19 \\
\hline Reading (all) & 180 & 40.1 & 21.87 & 14 & 76 \\
\hline High imageability words & 140 & 25 & 9.02 & 12 & 39 \\
\hline Low imageability words & 140 & 13.1 & 11.21 & 2 & 33 \\
\hline Picture naming & $/ 64$ & 34.9 & 13.40 & 7 & 54 \\
\hline Spoken word-picture matching & $/ 64$ & 61.8 & 2.53 & 56 & 64 \\
\hline Pyramid and Palm Trees test & 152 & 47.2 & 2.78 & 43 & 51 \\
\hline Rhyme judgement & $/ 48$ & 25.5 & 12.24 & 14 & 48 \\
\hline Rhyme production & 124 & 5.4 & 5.23 & 0 & 16 \\
\hline Phoneme segmentation ${ }^{\mathrm{b}}$ & 196 & 26.8 & 22.22 & 3 & 61 \\
\hline
\end{tabular}

a Test for the Reception of Grammar.

b $5 / 10$ Patients were unable to complete this task.

task used the same word and non-word items but only required production in the past-tense form. Each stem (present tense) was presented in a simple written sentence frame pair (e.g., Today I walk. Yesterday I ...) which was also read aloud by the examiner. The patients were asked to complete the second sentence by providing the past-tense form of the target verb. The profound non-fluent speech of these patients meant that these three production tasks required considerable effort from the participants to complete the tests. Consequently, the three tests were spread across eight testing sessions, with tasks and different conditions counterbalanced across sessions. All 10 patients were able to complete the repetition and reading tests but only five were able to finish the more demanding sentence completion task (patients: IB, DE, MB, GN, and JL).

The responses made by the patients were recorded, transcribed, and scored. The errors were then classified using two schemes-one for reading and repetition and another for sentence completion. For reading and repetition the following categories were used: responses that were phonologically near the target and were real words; responses that were phonologically near the target and were non-words; responses that were phonolog- ically distant from the target (typically these were non-word responses); omission of the -d/-t inflection; omission (i.e. no response); and other error types that occurred too rarely to form sensible bins (e.g., semantic errors; perseverative errors; addition of -ing or -s). To be classified as a close rather than distant phonological error a response had to preserve at least half of the target phonemes. The error scheme for the sentence completion task was based on the same classifications with additional categories to reflect past tense transformation-related errors, in particular a failure to do any transformation (e.g. Today I walk. Yesterday I. .. "walk").

\section{Results}

Fig. 1 shows the patients' mean error rate for past tense real verbs across the three production tasks. The results from this large set of unmatched regular and irregular verbs largely replicates the patients' performance on the screening materials: that is, despite very high error rates on both regular and irregular, the patients made relatively more errors to regular than 


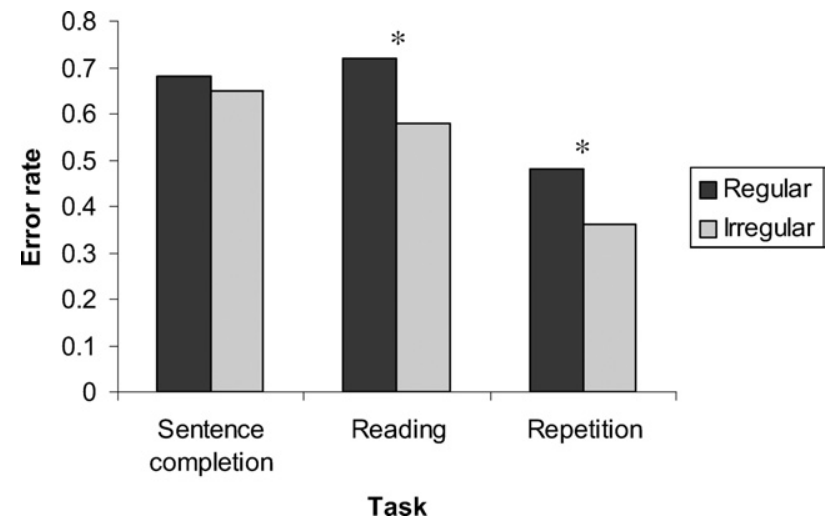

Fig. 1. Mean error rate for real verb targets. Asterisk denotes significant difference between regular and irregular verbs. Sentence completion data are derived from five patients. Reading and repetition data are based on the full 10 patients.

irregular real verb targets, a difference that was significant for reading $[t(9)=4.5, p=.002]$ and repetition $[t(9)=4.8, p=.001]$. A trend for the same pattern in the sentence completion data was not statistically significant. It should be recalled that only $5 / 10$ of the patients were able to perform the full sentence completion task, which reduces the power to detect a reliable difference and also means that data come mainly from the milder cases.

Although many different possible error types were included in the coding scheme (see Section 2), the patients produced four main types of errors: omission of $-\mathrm{d} /-\mathrm{t}$; phonologically close real word responses; phonologically close non-word responses; and phonologically distant responses. The rates of these errors (as a proportion of total errors) for real regular and irregular target verbs in reading and repetition are shown in Figs. 2A and B, respectively. The distribution of errors was very similar for these two tasks. As one might expect, omission of the inflection $-d$ or $-t$ was significantly greater for regular than irregular verbs [reading: $t(9)=3.5, p=.006$; repetition: $t(9)=4.3, p=.002]$. The opposite pattern was true for phonologically related real word responses, the rate of which was significantly higher for irregular than regular verbs [reading: $t(9)=5.0, p=.001$; repetition: $t(9)=4.6, p=.001]$. A similar pattern was also observed for the phonologically related non-word errors, although the difference was only statistically significant in the reading data [reading: $t(9)=2.7, p=.02$; repetition: $t(9)=1.7, p=.12]$.

Whereas the dual-mechanism account would clearly expect the omission of $-\mathrm{d} / \mathrm{-t}$ inflections from regular verbs, predictions from this account about types and rates of other errors do not seem obvious. In particular, the high rate of phonologically related word and nonword errors to irregular verbs does not seem to have a clear explanation in the dual-mechanism account. These errors are consistent, however, with the generalised pho-
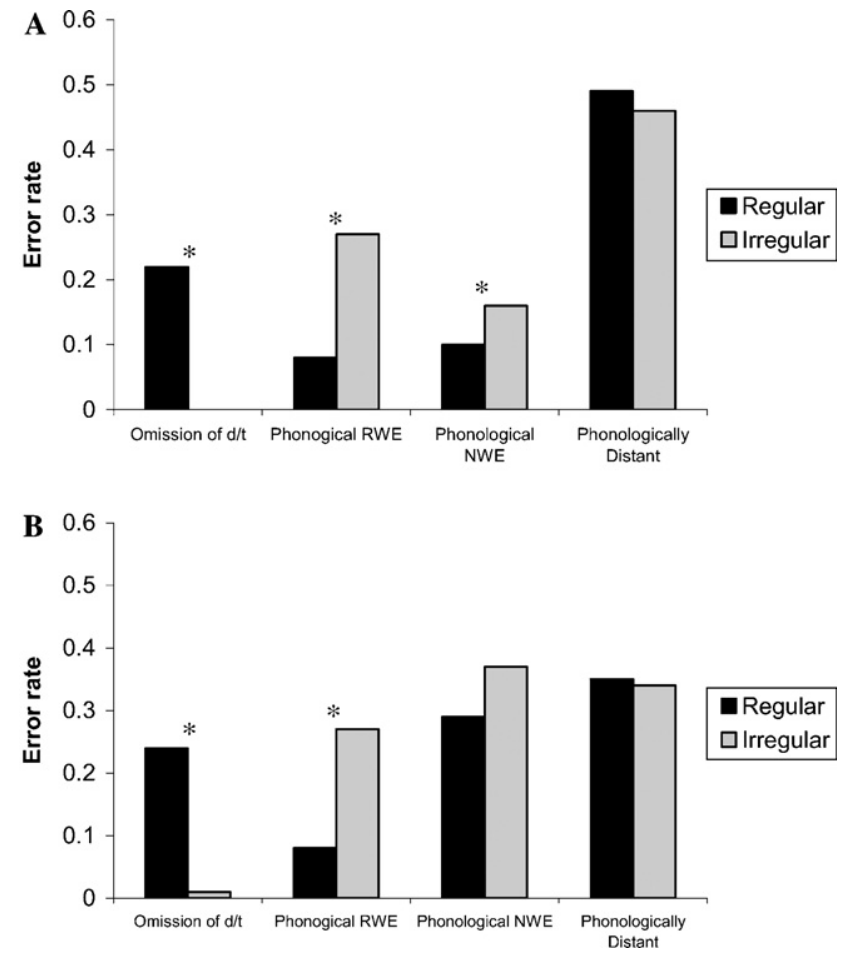

Fig. 2. (A) Distribution of error types in reading. (B) Distribution of error types in repetition. Asterisk denotes significant difference between regular and irregular verbs. RWE, real word error. NWE, non-word error. Phonological errors share at least half of their phonemes with the target word, phonologically distant errors are those that fall below this criterion.

nological impairment hypothesis. First, it is important to note that the four predominant error types produced by the patients were all (with varying approximations to the target) phonologically related to the correct response - as would be expected of patients with a phonological impairment. Second, while the omission of $-\mathrm{d} /-\mathrm{t}$ corresponds in morphological terms to a failure to inflect the stem, these responses can also be classified as close phonological real word errors. Indeed, this pattern - the cross-over interaction of regularity and error type (-d/-t omission vs. other phonological real word error) - could simply reflect the differential opportunity for making this particular type of phonological real word error on regular verbs. When omissions of $-\mathrm{d} /-\mathrm{t}$ are combined into the more general category of close phonological RWEs (Figs. 3A and B), there is no longer any significant differences in the rate of such errors for regular and irregular verbs [reading: $t(9)=0.72, p=.5$; repetition: $t(9)=0.5, p=.63]$. The most appropriate summary, therefore, would appear to be that the patients made a mixture of phonologically close and distant errors in repetition and reading. Although it is possible to extract one type of phonological real word error because it also corresponds to a morphological error (omission of $-\mathrm{d} / \mathrm{t}$ ) ), this produces a correspondingly higher rate of the remaining phonological real word errors for irregular verbs. 
This issue was tested further by investigating the rates and types of errors produced to non-word targets. The patients' performance for real verbs and phonologically matched non-words is shown in Fig. 4. Error rates in both reading and repetition were significantly greater
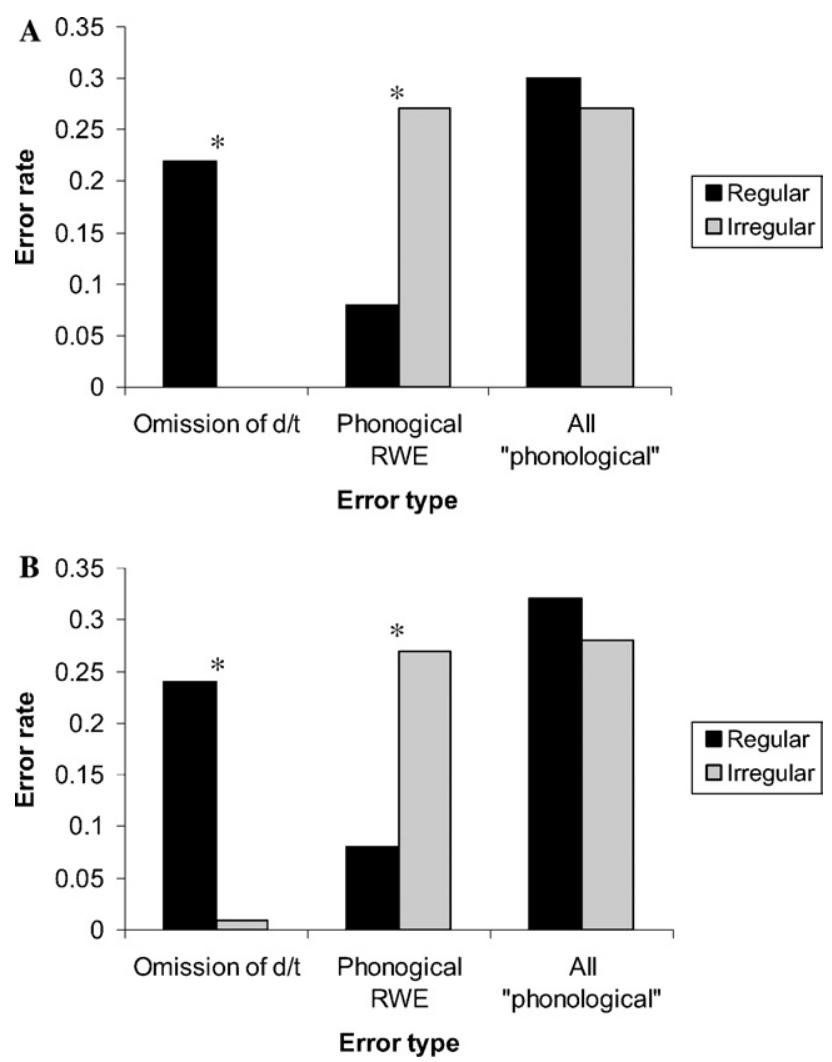

Fig. 3. (A) Comparison of different closely related phonological errors in reading. (B) Comparison of different closely related phonological errors in repetition. Asterisk denotes significant difference between regular and irregular verbs. RWE, real word error. NWE, non-word error, "All phonological" - combination of close phonologically related real word errors and $-\mathrm{d} /$-t omission errors.

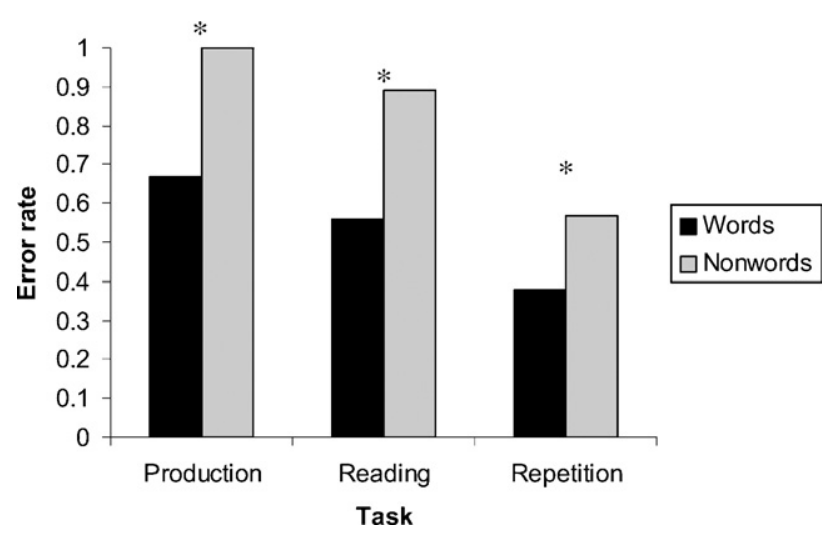

Fig. 4. Mean error rates for real verbs and non-words. Asterisk denotes significant difference between real verbs and non-words. Sentence completion data are derived from five patients. Reading and repetition data are based on the full 10 patients. for non-word than real verb targets $[\mathrm{F}(1,9)=84.1, p<$ $.001]$, and same pattern characterised sentence completion for the five patients who were able to complete this task. The rates of different error types for reading and repetition are shown in Figs. 5A and B, respectively, separately for word and non-word targets but collapsed across regular and irregular forms. In reading and repetition, the omission of $-\mathrm{d} / \mathrm{-t}$ was significantly more likely for real verb than non-word targets [reading: $t(9)=3.5$, $p=.006$; repetition: $t(9)=4.5, p=.001$ ] whilst the opposite was true for the rate of phonologically distant responses [reading: $t(9)=3.1, p=.01$; repetition: $t(9)=$ $4.45, p=.002]$. In repetition the patients also produced a significantly greater rate of phonological real word errors to non-words than real verbs $[t(9)=3.2$, $p=.01]$. If taken at face value, these results might appear to conflict with the generalised phonological impairment hypothesis, from which one might expect the rate of $-\mathrm{d} /$-t omissions to be equivalent for non-word and real verb targets - especially given that the nonwords were created from the real verbs by substituting one of the stem phonemes. Focussing on this specific error type, however, overlooks a more general influence on these patients' performance. Patients with phonological impairment generally find non-words particularly difficult to process (Patterson \& Marcel, 1992). In this group of patients, the lexicality effect can be seen in their
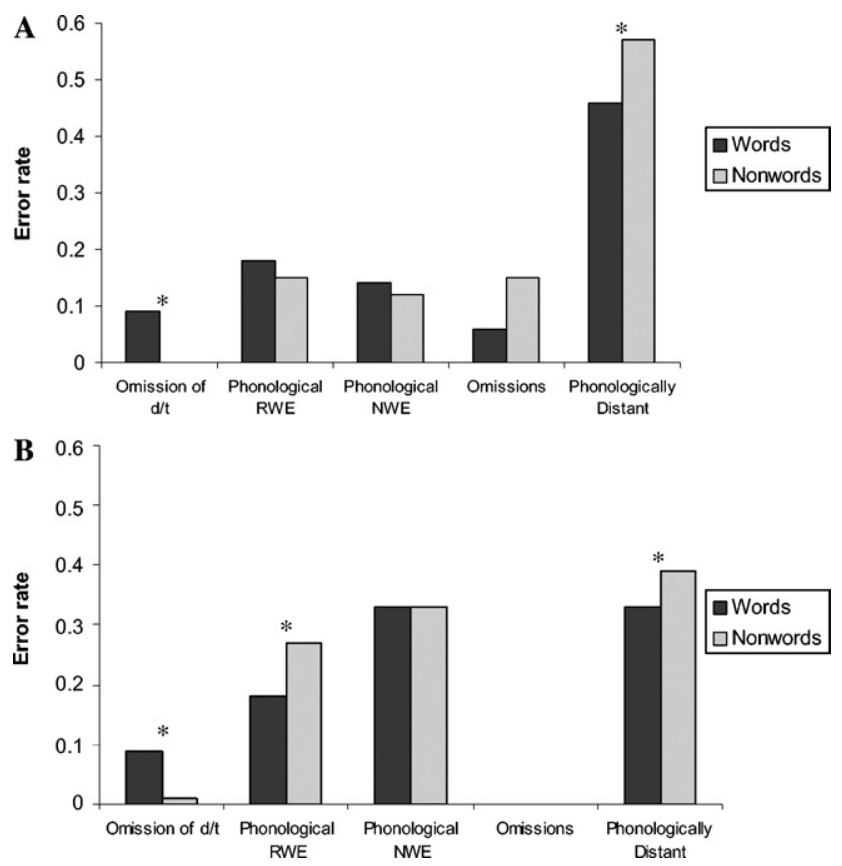

Fig. 5. (A) Distribution of error types for word and non-words in reading. (B) Distribution of error types for word and non-words in repetition. Asterisk denotes significant difference between real verbs and non-words. RWE, real word error. NWE, non-word error. Phonological errors share at least half of their phonemes with the target word, phonologically distant errors are those that fall below this criterion. 
relative error rates to words vs. non-words in all three production tasks (see Fig. 4). Figs. 5A and B demonstrate that the distribution of errors shifts when the patients were confronted with non-words. Specifically, the patients were less likely to respond with a real word response (combining - $\mathrm{d} /$-t omissions and phonological real word errors) and more likely to produce a distant phonological error or, in the case of reading, even to fail to respond altogether (omission errors).

Overall, there are three severity/difficulty dimensions along which the patients' error distribution shifted from close to distantly related responses. The first dimension is item difficulty: non-words, which were particularly challenging for these patients, yielded a higher rate of more distant errors. The second dimension is task diffculty: reading, which was more challenging than repetition (see Fig. 1), yielded a higher rate of phonologically distant errors $[t(9)=3.6, p=.006]$. The final dimension is patient severity: Figs. $6 \mathrm{~A}$ and $\mathrm{B}$ show scatterplots of patient severity (overall error rate) against the specific error rates for closely related vs. distant errors. In repetition (see Fig. 6B), there was a strong positive correla-
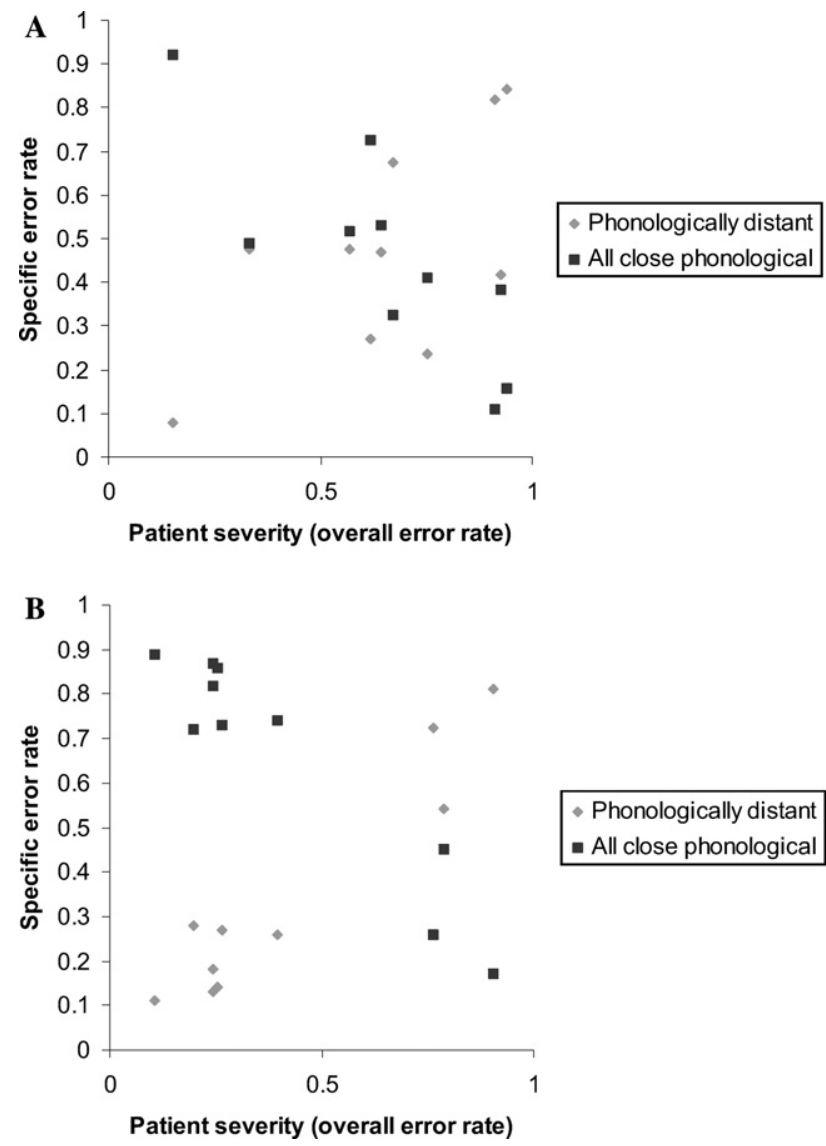

Fig. 6. (A) Relationship between patient severity and phonological error rate in reading. (B) Relationship between patient severity and phonological error rate in repetition. Patient severity was measure in terms of their overall averaged performance on the three verb tasks (reading, repetition, and sentence completion). A high severity rate corresponds to very poor performance on these three tests. tion for distant errors (the severest patients produced the greatest rate of distant errors: $r=.95, p<.001$ ) and negative correlation for closely related phonological errors $(r=-.95, p<.001)$. The same pattern was also observed in reading (patient severity was positively correlated with the rate of distant errors, $r=.63, p=.05$; and was negatively correlated with closely related responses, $r=-.82, p=.004$ ). So although the generalised phonological impairment hypothesis does predict a similar rate of phonologically based omissions of $-\mathrm{d} / \mathrm{t}$ for regular verbs and matched non-words, this prediction has to be evaluated in the context of performance that is consistently and strongly modulated by these three severity/difficulty-related factors. Specifically, when word-forms become difficult and/or the patients' disorder becomes severe, they cannot manage any close approximation to the target, whether an inflection-less stem or another phonologically related real word.

Analyses of the patients' errors on the sentence completion task are limited by the fact that only $5 / 10$ patients, generally the milder ones, were able to complete the task. The errors made by these five patients were dominated by two types: no change errors (i.e., repetition of the uninflected stem that they had just heard in the first part of the sentence pair) and distant phonological errors. The rates of these two error types for the different word forms are shown in Fig. 7. The most striking aspect of these patients' performance is that, as for reading and repetition, the difficulty of the target items shifted the distribution of errors: patients made more no change errors to real verbs: $[t(4)=3.9, p=.02]$ and more distant phonological errors to non-words $[t(4)=$ $2.6, p=.06]$. There was no significant influence of regularity on this pattern.

\subsection{What do the patients' errors tell us about the quality of their phonological impairment?}

The first set of analyses reported above revealed that errors in reading, repeating and generating the past-tense

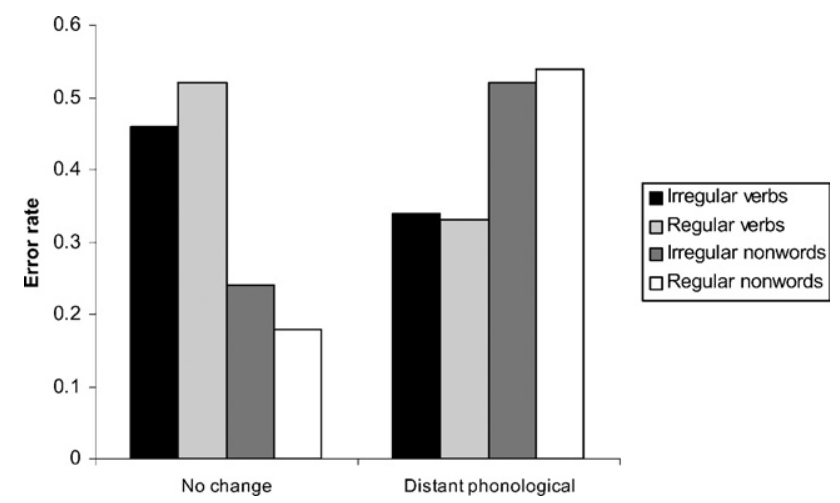

Fig. 7. Rate of 'no change' and distant phonological errors in sentence completion. 
forms of regular, irregular, and nonce verbs were predominantly phonological in nature, as one would expect from patients with a generalised phonological impairment. The additional analyses reported below were conducted in an attempt to reveal more about the nature of this phonological impairment and, in particular, what features were germane to the patients' relatively greater inaccuracy for regularly inflected verbs (a difference that was significant for reading and repetition, see Fig. 1). Initially all phonologically related errors were classified into five types: substitution (of 1 or more phonemes), deletion (ditto), addition (ditto), multiple (involving combinations of substitutions, deletions or additions), and other. The rates of these different types of phonological errors are shown in Fig. 8 for reading and repetition. In both tasks, phonological substitutions were more common than deletions [reading: $t(9)=3.6, p=.006$; repetition: $t(9)=9.2, p<.001]$ which, in turn, were more frequent than additions [reading: $t(9)=2.3, p=.05$; repetition: $t(9)=2.6, p=.03]$. The same errors were then reclassified according to whether the error was more or less phonologically complex than the target, based on the number of consonants in the onset and offset. Fig. 9 shows that although patients' phonological errors did sometimes result in a more complex form, the strong tendency was to make the form phonologically simpler [reading: $t(9)=2.5, p=.03$; repetition: $t(9)=2.6, p=.03$ ] .

The tendency of patients with Broca's aphasia to reduce the phonological complexity of words when they speak has been noted in other studies (Romani \& Calabrese, 1998; Romani, Olson, Semenza, \& Grana, 2002) as well as in general descriptions of this form of aphasia (Brown, 1972). When the present results are combined with those of other studies, it seems clear that phonological complexity is a critical factor in the patients' performance. They are severely impaired at producing the past-tense forms of both regular and irregular verbs, and to an equal degree once the two sets are matched for phonological complexity of the past-tense forms (Bird et al., 2003). In an unmatched set, regular past-

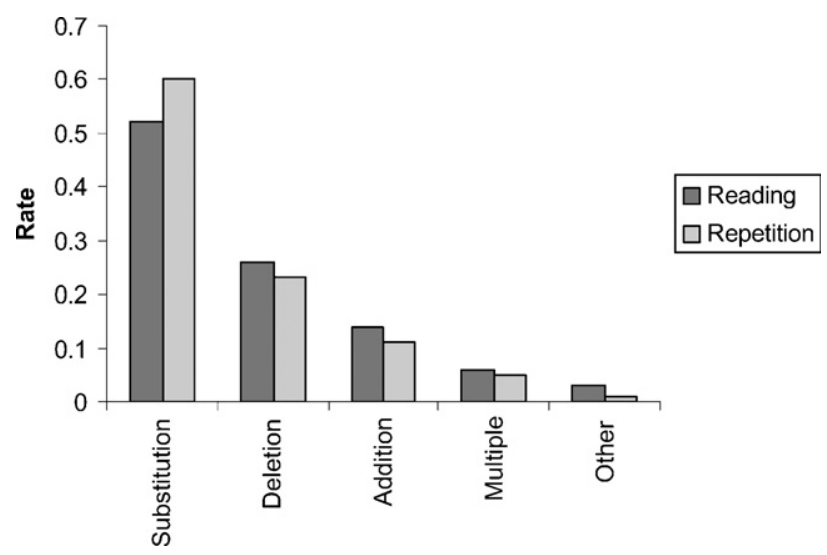

Fig. 8. Types of phonological errors for reading and repetition.

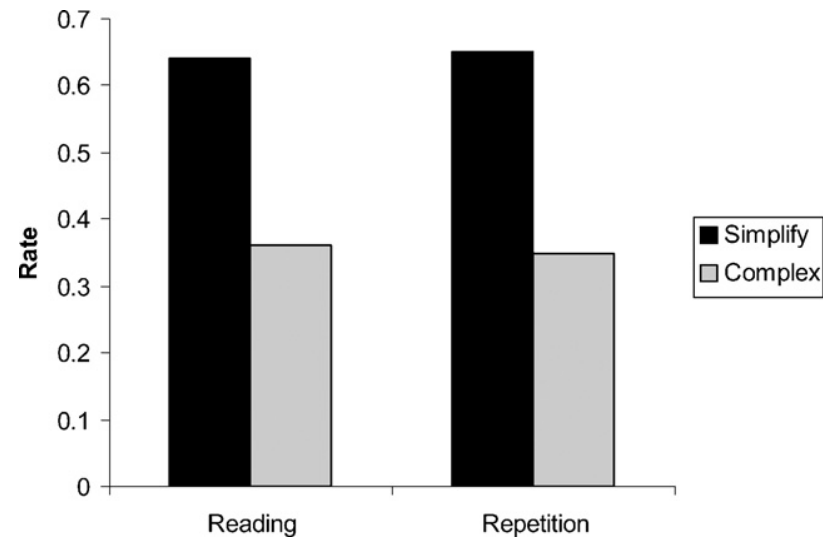

Fig. 9. Rate of phonological simplifications vs. more complex errors.

tense forms will on average be more phonologically complex than irregulars; therefore, the fact that the patients' impaired phonological system results in a strong tendency to reduce the complexity of spoken forms puts the regular forms at an inherent disadvantage. If complexity is a critical factor, then its influence should be observed at any position in the word form, not just on the regular inflection. To test this prediction, we conducted a vowel-centred position analysis on the patients' phonologically related errors (combined across all three tasks). Each target-error pairing was aligned at the vowel and the prevocalic, vowel, and postvocalic phonemes were compared for each position. As shown in Fig. 10, accuracy varied significantly across position $[F(1,6)=4.8, p=.001]$. The patients' incorrect responses were significantly more likely to preserve the target vowel than either onset or offset consonants [all pairwise comparisons between vowel and other positions were significant: $t(9)$ between 3.0 and $9.6, p$ between .01 and $<.001]$. There were no differences between onset and offset consonants, however: when the vowel position data were removed from the analysis there was no longer a significant effect of position on accuracy $[F(1,5)=1.6$, $p=.18]$. The vowel occupies a longer, more stable component of the spoken word which is likely to yield a

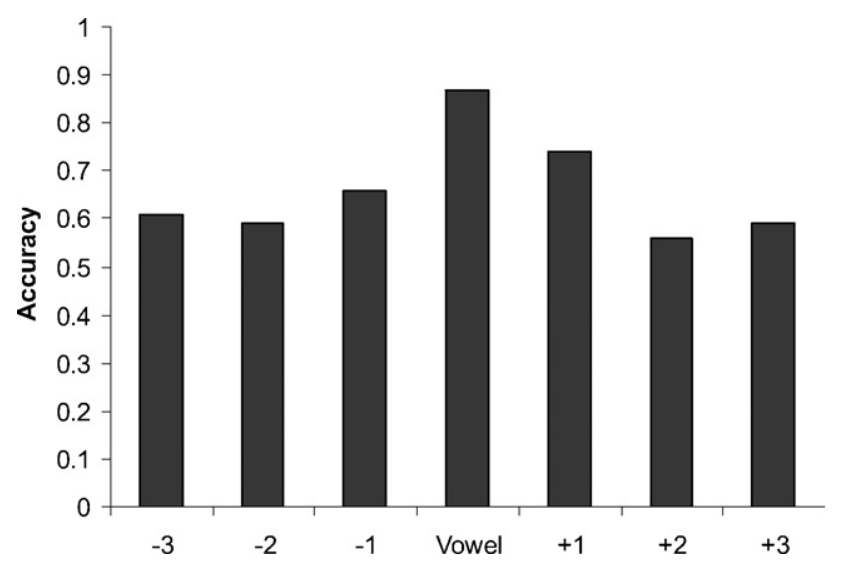

Fig. 10. Position analysis for phonologically related errors. 
more salient part of the phonological representation which, in turn, will likely be more robust to damage. In contrast, the transient nature of consonants and, in particular, stops will produce weak phonological representations (Hoeffner \& McClelland, 1993).

\section{General discussion}

This study was designed to extend the investigation previously reported by Bird et al. (2003) on 10 patients with Broca-type aphasia. The aim was to use the large corpus of errors produced by the patients in reading, repetition and sentence completion to test the notion that a generalised phonological impairment was central to the patients' language impairment and to reveal some of the qualities of this deficit. In particular, we were keen to understand those qualities that might explain the patients' relatively poorer performance on regular past tense verb forms observed in unmatched materials. As emphasised by McClelland and Patterson (2002), we do not contest the fact that these patients have a morphological and/or syntactic deficit that contributes to their severely impaired performance on both regular and irregular verbs. The manner in which our claim, and account, differs from the dual-mechanism account of Pinker and Ullman (2002) and Ullman et al. (1997) is that we explain the relatively higher error rate for regular past-tense forms as resulting from an underlying phonological, rather than a morphological, deficit.

The centrality of the patients' phonological impairment was reinforced by the fact that the majority of their errors in repetition, reading, and sentence completion could be classified as either close or distant phonological errors. In reading and repetition, there were significantly more inflectional (terminal $-\mathrm{d} / \mathrm{-t}$ ) omissions for regular than irregular verbs as one might expect from a dual mechanism account (Pinker, 1999; Ullman, 2001b; U11man et al., 1997). Our analysis suggests, however, that these 'inflectional' omissions were in fact a subset of the more general class of phonological real word errors, given that non-morphological, phonological real word errors were more prevalent for irregular than regular verbs; once the two error categories were combined, there was no remaining regularity effect. It would appear, therefore, that $-\mathrm{d} / \mathrm{-t}$ deletions simply reflected the greater opportunity for this type of phonological error in regular verbs while other types of phonological error occurred at a similar rate for irregular verbs. In sentence completion, the most common error types were no change (reproduction of the uninflected stem) and distant phonological responses.

The balance between closely related phonological errors and more distant responses in reading and repetition was found to vary along three different severity/ difficulty continua. When the items or tasks became harder (non-words $>$ words; sentence completion $>$ reading $>$ repetition), or the severity of the patients increased (as measured by overall error rate in these tasks), then the balance of the phonological errors shifted away from closely to more distantly-related responses. This shift in error type may explain why a significantly greater number of $-\mathrm{d} / \mathrm{-t}$ omission errors occurred in response to regular real verbs than to their non-word counterparts. Given that the non-words were constructed from the real verbs by substituting one of the stem phonemes, one might expect a generalised phonological impairment to lead to a similar pattern of errors for words and non-words. Patients with phonological impairment, however, find non-words particularly challenging, as reflected in this study by near-floor rates of overall success on non-word targets. In most cases, when an overt error (as opposed to no response) was produced, the non-word form was grossly distorted. In such circumstances, the resultant spoken form is very unlikely to fall into any type of closely related error category - including the omission of the $-\mathrm{d} /$-t inflection.

The results from this and other studies (Bird et al., 2003; Romani \& Calabrese, 1998; Romani et al., 2002) reinforce the notion that complexity is a key factor influencing the patients' phonological impairment. The patients' phonologically related errors were made up from a combination of substitutions, deletions, and additions, but with significantly more deletions than additions. These different phonological transformations most often resulted in a simplification of the phonological form. A subsequent position analysis showed that these phonological changes occurred across the word; there was no specific focus on the offset of words as one might expect from a morphologically specific deficit. Instead, the patients' performance was consistent with a generalised phonological impairment influenced by phonological complexity, suggesting that it is the increase in phonological complexity in regular pasttense forms like "typed" rather than the inflection itself that presents a challenge to the patients' impaired phonology.

In conclusion, these results add weight to the idea that a variety of language activities rely upon the integrity of a limited set of primary brain systems including semantic knowledge and phonology (Neville \& Bavelier, 1998; Patterson \& Lambon Ralph, 1999) as well as syntactic/morphological knowledge. Much of what is needed for past tense generation can be captured by the interaction between semantics and phonology (Joanisse \& Seidenberg, 1999; Rumelhart \& McClelland, 1986). Phonology is key to this transformation given that consistencies within irregular verbs are based on their phonological form and that the exact allomorph used to represent the regular inflection is phonologically constrained (McClelland \& Patterson, 2002). Syntactic/ morphological, as well as phonological, impairments, 
probably contribute to poor performance for all three types of verb (regular, irregular, and non-words); but we conclude that the extra deficit on regular inflections required for real regular verbs and nonce verbs can be explained solely in terms of phonological difficulties, without the rule-based impairment postulated by a dual-mechanism account.

\section{Acknowledgments}

This work was supported by a project grant from the Leverhulme Trust $(\mathrm{F} / 00120 / \mathrm{M})$ and a grant from the NIMH (MH64445). We are indebted to the continued support of the patients and their carers.

\section{References}

Bird, H., Lambon Ralph, M. A., Patterson, K., \& Hodges, J. R. (2000). The rise and fall of frequency and imageability: Noun and verb production in semantic dementia. Brain and Language, 73, 17-49.

Bird, H., Lambon Ralph, M. A., Seidenberg, M. S., McClelland, J. L., \& Patterson, K. (2003). Deficits in phonology and past tense morphology. Journal of Memory and Language, 48, 502-526.

Bishop, D. V. M. (1989). Test for the reception of grammar. London: Medical Research Council.

Bozeat, S., Lambon Ralph, M. A., Patterson, K., Garrard, P., \& Hodges, J. R. (2000). Non-verbal semantic impairment in semantic dementia. Neuropsychologia, 38, 1207-1215.

Brown, J. (1972). Aphasia, apraxia and agnosia. Springfield, IL: Charles C. Thomas.

Burzio, L. (2002). Missing players: Phonology and the past-tense debate. Lingua, 112, 157-199.

Goodglass, H., \& Kaplan, E. (1983). The assessment of aphasia and related disorders. Philadelphia: Lea \& Febiger.

Hodges, J. R., Patterson, K., Oxbury, S., \& Funnell, E. (1992). Semantic dementia: Progressive fluent aphasia with temporal lobe atrophy. Brain, 115, 1783-1806.

Hoeffner, J., \& McClelland, J. L. (1993). Can a perceptual processing deficit explain the impairment of inflectional morphology in developmental dysphasia? A computational investigation. In E. Clark (Ed.), Proceedings of the twenty-fifth annual child language research forum (pp. 38-49). Palo Alto, CA: Stanford University.

Howard, D., \& Patterson, K. (1992). The Pyramids and Palm Trees Test: A test of semantic access from words and pictures. Bury St. Edmunds: Thames Valley Test Company.

Joanisse, M. F., \& Seidenberg, M. S. (1999). Impairments in verb morphology after brain injury: A connectionist model. Proceedings of the National Academy of Sciences of the United States of America, 96, 7592-7597.
Marslen-Wilson, W. D., \& Tyler, L. K. (1997). Dissociating types of mental computation. Nature, 387, 592-594.

Marslen-Wilson, W. D., \& Tyler, L. K. (1998). Rules, representations, and the English past tense. Trends in Cognitive Sciences, 2, 428-435.

McClelland, J. L., \& Patterson, K. (2002). Rules or connections in past-tense inflections: What does the evidence rule out?. Trends in Cognitive Sciences, 6, 465-472.

Neville, H. J., \& Bavelier, D. (1998). Neural organization and plasticity of language. Current Opinion in Neurobiology, 8, 254-258.

Patterson, K., \& Lambon Ralph, M. A. (1999). Selective disorders of reading?. Current Opinion in Neurobiology, 9, 235-239.

Patterson, K., Lambon Ralph, M. A., Hodges, J. R., \& McClelland, J. L. (2001). Deficits in irregular past-tense verb morphology associated with degraded semantic knowledge. Neuropsychologia, 39, 709-724.

Patterson, K., \& Marcel, A. (1992). Phonological ALEXIA or PHONOLOGICAL alexia?. In J. Alegria, D. Holender, J. Juncas de Morais, \& M. Radeau (Eds.), Analytic approaches to human cognition. Amsterdam: North-Holland.

Pinker, S. (1999). Words and rules: The ingredients of language. London: Weidenfeld \& Nicolson.

Pinker, S., \& Ullman, M. T. (2002). The past and future of the past tense. Trends in Cognitive Sciences, 6, 456-463.

Plunkett, K., \& Nakisa, R. C. (1997). A connectionist model of the Arabic plural system. Language and Cognitive Processes, 12, 807-836.

Romani, C., \& Calabrese, A. (1998). Syllabic constraints in the phonological errors of an aphasic patient. Brain and Language, 64, $83-121$

Romani, C., Olson, A., Semenza, C., \& Grana, A. (2002). Patterns of phonological errors as a function of a phonological versus articulatory locus of impairment. Cortex, 38, 541-567.

Rumelhart, D. E., McClelland, J. L. (1986). On learning the past tense of English verbs. In J. L. McClelland, D. E. Rumelhart \& a. t. P. R. Group (Eds.), Parallel distributed processing: Explorations in the microstructure of cognition, Vol. 2, Psychological and Biological Models. Cambridge, MA: MIT Press.

Tyler, L. K., de Mornay Davies, P., Anokhina, R., Longworth, C., Randall, B., \& Marslen-Wilson, W. D. (2002a). Dissociations in processing past tense morphology: Neuropathology and behavioural studies. Journal of Cognitive Neuroscience, 14, 79-94.

Tyler, L. K., Randall, B., \& Marslen-Wilson, W. D. (2002b). Phonology and neuropsychology of the English past tense. Neuropsychologia, 1373, 1-13.

Ullman, M. T. (2001a). The declarative/procedural model of lexicon and grammar. Journal of Psycholinguistic Research, 30, 3769.

Ullman, M. T. (2001b). A neurocognitive perspective on language: The declarative/procedural model. Neuroscience, 2, 717-726.

Ullman, M. T., Corkin, S., Coppola, M., Hickok, G., Growdon, J. H., Koroshetz, W. J., \& Pinker, S. (1997). A neural dissociation within language: Evidence that the mental dictionary is part of declarative memory, and that grammatical rules are processed by the procedural system. Journal of Cognitive Neuroscience, 9, 266-276. 